\title{
Comparing AGN broad and narrow line regions
}

\section{Nicola Bennert ${ }^{1}$, Heino Falcke ${ }^{2}$, Yuri Shchekinov ${ }^{3}$ and Andrew S. Wilson ${ }^{4}$}

\author{
${ }^{1}$ AIRUB, Universitätsstraße 150, 44780 Bochum, Germany \\ ${ }^{2}$ ASTRON, P.O. Box 2, 7990 AA Dwingeloo, The Netherlands \\ ${ }^{3}$ Rostov State University, Department of Physics, 344090 Rostov on Don, Russia \\ ${ }^{4}$ Astronomy Department, University of Maryland, College Park, MD 20742-2421, USA
}

\begin{abstract}
We compare recent HST observations of Seyfert and quasar NLRs and find that type-2 AGNs follow a relation consistent with that expected for a distribution of gas ionized by a central source $R_{\mathrm{NLR}, 2} \propto L^{0.32 \pm 0.05}$, while type- 1 objects are fit with a steeper slope of $0.55 \pm 0.05$. The latter is comparable to the scaling found for the BLR size with continuum luminosity (slope: $0.5-0.7$ ). Therefore, we investigate what we can learn about the BLR size if the NLR size is only determined by the AGN luminosity. We find that NLR and BLR size are related linearly following $R_{\mathrm{BLR}} \propto R_{\mathrm{NLR}, 1}^{0.88 \pm 0.1}$. This relation can be used to estimate $\mathrm{BH}$ masses.
\end{abstract}

\section{Summary}

Sizes and morphologies of broad- and narrow-line regions (BLRs, NLRs) provide an ideal probe of the distribution of dust and gas in the central parts of AGNs. However, until today, even very basic issues remain open: What determines the size and structure of these emission-line regions? Do they grow with luminosity, and if so, how? For the NLR, the latter was investigated for the first time by Bennert et al. (2002) for a sample of seven radio-quiet PG quasars observed in the [OIII] $\lambda 5007$ line with HST. We found a NLR size-luminosity relation $R_{\mathrm{NLR}} \propto L_{[\mathrm{OIII}]}^{0.5}$ when including a sample of seven Seyfert-2 galaxies (Falcke et al. 1998). This result is remarkable if it implies that it is not the Strömgren radius that limits the NLR but an apparent threshold in ionizing flux, which can be expressed by a constant product of ionization parameter and density at the rim of the NLR. It is not clear whether this new relation will hold at all luminosities and redshifts. If, for example, the luminosity-scaled size of the NLR becomes larger than the size of the galaxy, the relation may flatten as the emission lines fade out and disappear (see Netzer 2004, these proceedings). This may be evident already in some spectral line studies of Two Degree Field quasars (Croom et al. 2002).

More recently, Schmitt et al. (2003) studied the extended [OIII] emission in a sample of 60 Seyfert galaxies, finding that the NLR size-luminosity relation follows a simple Strömgren law $R_{\mathrm{NLR}} \propto L_{[\mathrm{OIII}]}^{0.33}$. What causes the apparent different slopes for our quasardominated sample (0.5) and their Seyfert sample (0.33): simple statistical uncertainty or selection effects? Are the NLRs of Seyferts and quasars intrinsically different? Or is there a difference in the NLR size-luminosity relation between type- 1 and type- 2 objects? Puzzled by these questions, we compared the two samples and calculated a fit to all type -1 and type -2 AGNs separately: While type -1 objects follow the relation $R_{\mathrm{NLR}, 1} \propto$ $L^{0.55 \pm 0.05}$, type $2 \mathrm{~s}$ can be fit by approximately a Strömgren-law $R_{\mathrm{NLR}, 2} \propto L^{0.32 \pm 0.05}$ (Fig. 1 , left). This result explains the different slopes found by the two groups: In the Bennert et al. (2002) sample, basically type-1 quasars define the slope, whereas the slope of the 

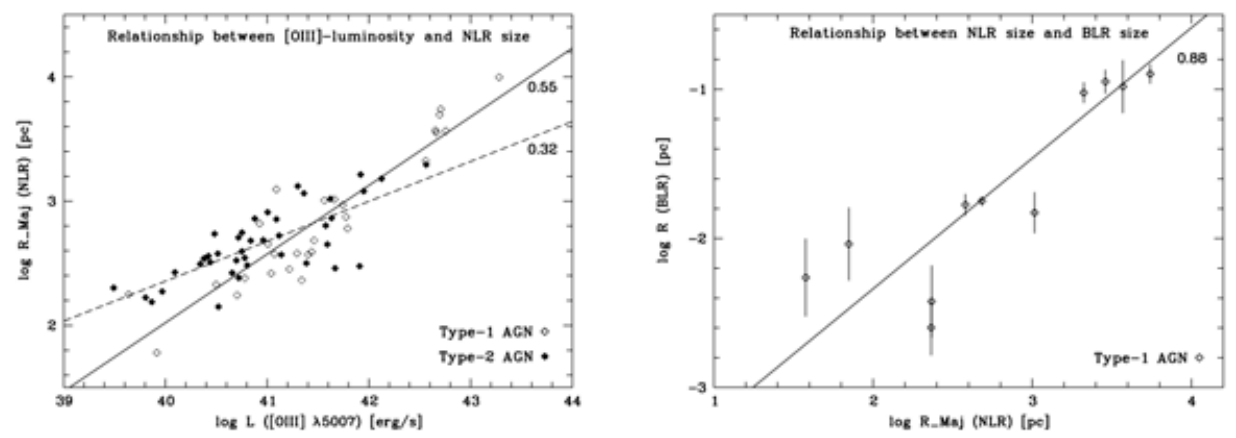

Figure 1. Left: Radius of the NLR versus the emission-line luminosity in [OIII] on logarithmic scales. The solid line represents the fit to type-1 AGNs $\left(R_{\text {Maj }}(\mathrm{NLR}, 1) \propto L_{[\mathrm{OIII}]}^{0.55 \pm 0.05}\right)$, the dashed line the fit to type -2 AGNs $\left(R_{\mathrm{Maj}}(\mathrm{NLR}, 2) \propto L_{[\mathrm{OIII}]}^{0.32 \pm 0.05}\right)$. Right: Distribution of NLR and BLR radii on logarithmic scales. The error bars indicate the uncertainty in defining the BLR radius. The solid line represents the fit corresponding to $R(\mathrm{BLR}) \propto R_{\mathrm{Maj}}(\mathrm{NLR}, 1)^{0.88 \pm 0.1}$.

Schmitt et al. (2003) sample is dominated by Seyfert 2s, which outnumber the Seyfert 1 s by a factor of 1.4 .

As the BLR is too compact to derive its size directly, reverberation mapping techniques are used (e.g. Wandel et al. 1999; Kaspi et al. 2000). While Kaspi et al. (2000) report a BLR size-luminosity relation of $R_{\mathrm{BLR}} \propto L^{0.7}$, McLure \& Jarvis (2002) find $R_{\mathrm{BLR}} \propto L^{0.5}$. If the NLR size is only determined by the AGN luminosity, it is of interest what we can learn about the BLR size and BH mass. We compared all measured BLR sizes with their corresponding NLR sizes. Unfortunately, the overlap is rather poor and leaves us with 11 objects. Applying a weighted linear least-squares fit, we find that the sizes of NLR and BLR are proportional: $R_{\mathrm{BLR}} \propto R_{\mathrm{NLR}, 1}^{0.88 \pm 0.1}$ (Fig. 1, right).

Since deriving BLR sizes is very time consuming, relationships with luminosity or NLR size can be extremely useful for estimating BH masses. Given the NLR size (either directly measured or derived from the [OIII] luminosity), the BLR size can be estimated, which, assuming Keplerian motions, yields $M_{\mathrm{BH}}$ :

$$
M_{\mathrm{BH}}=\left(10^{5.22} M_{\odot}\right) v_{3000}^{2} R_{\mathrm{NLR}, 1}^{0.88} \quad \text { or } \quad M_{\mathrm{BH}}=\left(10^{8.83} M_{\odot}\right) v_{3000}^{2} L_{44,[\mathrm{OIII}]}^{0.48}
$$

$\left(v_{3000}=\mathrm{FWHM}(\mathrm{H} \beta) / 3000 \mathrm{~km} \mathrm{~s}^{-1}, R_{\mathrm{NLR}, 1}\right.$ in parsec, $L_{44,[\mathrm{OIII}]}=[\mathrm{OIII}]$ luminosity $/ 10^{44}$ $\left.\mathrm{erg} \mathrm{s}^{-1}\right)$. The latter equation is similar to $M_{\mathrm{BH}} \propto L_{5100 \AA}^{0.5}$ found by Shields et al. (2003).

\section{Acknowledgements}

In memoriam Prof. Hartmut Schulz, deceased in August 2003. N.B. remembers him gratefully for having been her "Doktorvater" in the truest sense of the word. The astronomical community has lost a wonderful colleague and a truly independent mind.

\section{References}

Bennert, N., Falcke, H., Schulz, H., Wilson, A. S., \& Wills, B. J. 2002, ApJ, 574, 105

Croom, S. M., et al. 2002, MNRAS, 337, 275

Falcke, H., Wilson, A. S., \& Simpson, C. 1998, ApJ, 502, 199

Kaspi, S., et al. 2000, ApJ, 533, 631

McLure, R. J., \& Jarvis, M. J. 2002, MNRAS, 337, 109

Schmitt, H. R., et al. 2003, ApJS, 597, 768

Shields, G. A., et al. 2003, ApJ, 583, 124

Wandel, A., Peterson, B. M., \& Malkan, M. A. 1999, ApJ, 526, 579 